Pacific Journal of Mathematics

ON THE DEGENERACY OF A SPECTRAL SEQUENCE 


\title{
ON THE DEGENERACY OF A SPECTRAL SEQUENCE ASSOCIATED TO NORMAL GROSSINGS
}

\author{
Gerald LeONARD GoRDON
}

\begin{abstract}
Let $W$ be a complex analytic manifold and $V$ a divisor with normal crossings, and consider the Leray spectral sequence associated to the inclusion map of $W-V$ into $W$. We give two homological reformulations for any of the $d_{r}^{p, q}$ to be the zero map for $r \geqq 2$. These conditions are shown to be satisfied if $W$ is compact Kähler, but it is easy to give examples when it does not degenerate at $E_{3}$ if $W$ is only a differentiable manifold. The nondegeneracy at $E_{3}$ for arbitrary $V$ in a compact Kähler manifold is interpreted in terms of reiterated residues.
\end{abstract}

\section{Introduction.}

1.1. Let $j: W-V \subset W$ be the inclusion. When $W$ is a projective, algebraic manifold and $V$ is a divisor with normal crossings, then Deligne [2] in the course of defining his mixed Hodge structure in $W-V$ shows that the Leray spectral sequence associated to $j$ degenerates at $E_{3}$, i.e., $E_{3}^{p, q} \cong E_{\infty}^{p, q}$ for all $p, q$. Griffiths-Schmid [7] give another proof of the degeneracy at $E_{3}$, when $W$ is a compact Kähler manifold and $V$ again has normal crossings.

In this article, we let $W$ be a complex analytic manifold and $V$ a divisor with normal crossings. In Theorem 2.3 we then give two homological reformulations for any of the $d_{r}^{p, q}$ to be the zero map. It is easy to give differentiable examples where these topological conditions are not satisfied. However, we know of no examples of non-Kähler compact complex manifolds where these conditions fail. The degeneracy at $E_{3}$ might be true for surfaces but we feel it is not true in dimensions greater than two.

If $W$ is compact Kähler, then these homological conditions are satisfied for divisors with normal crossings. However, if one allows arbitrary singularities on $V$, then one can give examples when the homological conditions of (2.3) are not satisfied; so that the general position is needed, although the abutment of the spectral sequence is the same when one resolves the singularities to normal crossings. This phenomenon is interpreted in terms of iterated residues in (3.2.1).

As far as notation goes, $H^{*}(X)$ or $H_{*}(X)$ will always mean coefficients in some fixed field. If $W$ is noncompact, the homology or cohomology can either be with compact or closed support. By closed support in $W-V$, we shall mean support closed in $W-V$ and closed in $W$, cf., Fotiadi, et. al [3, Part III]. 
2. A homological reformulation for degeneracy.

2.1. Suppose $W$ is a complex manifold of complex dimension $n$ and $V$ is a divisor with normal crossings. We suppose $V_{i}, i \in I$ are the nonsingular components of $V$ and we set $V_{i_{1} \cdots i_{k}}=V_{i_{1}} \cap \cdots \cap V_{i_{k}}$, which is a submanifold of complex dimension $n-k$ or the empty set. We let $M_{k}$ denote the $k$-tuple points of $V$ (i.e., $M_{k}=U\left(V_{i_{1} \cdots i_{k}}-\right.$ $\left.\bigcup_{i_{k+1}} V_{i_{1} \cdots i_{k} i_{k+1}}\right)$ ) and $\bar{M}_{k}$ denotes the closure of $M_{k}$. Set $M_{0}=W-V$.

Then in Gordon [4, p. 133] a subgroup of $H_{p}\left(\bar{M}_{q}\right)$ is defined, called the tubular cycles, and denoted by $H_{p}\left(\bar{M}_{q}\right)_{\Delta}$, such that one has the tube over cycle map $\tau_{q}: H_{p}\left(\bar{M}_{q}\right)_{4} \rightarrow H_{p+1}\left(M_{q-1}\right)$. One also has the Gysin mapping $\widetilde{\tau}_{q}: H_{p}\left(M_{q}\right) \rightarrow H_{p+1}\left(M_{q-1}\right)$, cf., [4, p. 134].

\subsubsection{DEFINITION.}

$$
H_{p}(V)_{\Delta}=H_{p}\left(\bar{M}_{1}\right)_{)_{q=2}}^{p} \tilde{\tau}_{2} \cdots \tilde{\tau}_{q-1} \tau_{q} H_{p-q+1}\left(\bar{M}_{q}\right)_{\Delta} .
$$

If we let $\tau$ denote $\tau_{1}$ on the first summand and $\tilde{\tau}_{1}$ on the last $p$ summands of (2.1.1), then Gordon [4, p. 143] shows:

2.1.2. The following is exact:

$$
H_{p+2}(W) \stackrel{I}{\longrightarrow} H_{p}(V)_{\Delta} \stackrel{\tau}{\longrightarrow} H_{p+1}(W-V)
$$

where $I$ is transverse intersection with $V$.

2.2. We are now going to define the notion of absolutely relative class of degree $k$.

Suppose $V_{i}, i \in I^{\prime}$ are complex manifolds in general position and suppose we have $\gamma_{p} \in H_{p}\left(\cup V_{i}\right)$. Let $\gamma_{p} \cap V_{i}=\gamma_{p, i} \in H_{p}\left(V_{i}, \cup_{j, j \in I_{I}} V_{i j}\right)$ and $j_{i}: V_{i} \subset\left(V_{i}, \mathrm{U}_{j, j \in I^{\prime}}, V_{i j}\right)$ be the inclusion. (Recall, $V_{i j}=V_{i} \cap V_{j}$.) Finally, let Hom: $H_{p}\left(V_{i}, \cup_{j} V_{i j}\right) \stackrel{\sim}{\rightarrow} H^{p}\left(V_{i}, \bigcup_{j} V_{i j}\right)$ be vector space duality. The pairing can be thought of as being given by deRham's theorem via integration, where $H^{*}\left(V_{i}, \bigcup_{j} V_{i j}\right)$ can be represented by forms which vanish on $\mathrm{U}_{j} V_{i j}$, cf., Leray [9, Chapter 3]. If the $V_{i}$ are noncompact, then $H_{*}^{c}\left(V_{i}, \bigcup_{j} V_{i j}\right) \stackrel{\sim}{\rightarrow} H_{F}^{*}\left(V_{i}, \bigcup_{j} V_{i j}\right)$ where $c$ and $F$ denote compact and closed support respectively.

2.2.1. Definition. We say $\gamma_{p} \in H_{p}\left(\bigcup_{i \in I^{\prime}} V_{\imath}\right)$ is an absolutely relative class of $\bigcup_{i \in I^{\prime}}, V_{i}$ if

(i) $0 \neq \gamma_{p, i} \in H_{p}\left(V_{i}, \bigcup_{j} V_{i j}\right)$ for all $i \in I^{\prime}$.

(ii) $\left(j_{i}\right)^{*} \operatorname{Hom}\left(\gamma_{p, i}\right)=0$ for all $i \in I^{\prime}$.

Thus, suppose $V=V_{1} \cup V_{2}$ are two Riemann surfaces with two double points $P$ and $Q$ in common. Then a real line joining $P$ to 
$Q$ in $V_{i}$ for $i=1$ and 2 would represent an absolutely relative class if it did not wrap around a handle of $V_{i}$ in going from $P$ to $Q$.

Suppose $\gamma_{p}$ is an absolutely relative class of $\bigcup_{i \in I^{\prime}} V_{i}$. Then we have,

$$
0 \neq \partial_{*} \gamma_{p, i}=\sum_{j \in 1} \gamma_{p-1, i j} \in H_{p-1}\left(\mathbf{U}_{j} V_{i j}\right)
$$

2.2.2. Definition. $\gamma_{p}$ is an absolutely relative class of degree one of $U V_{i}$ if $\gamma_{p}$ is an absolutely relative class such that $\partial_{*} \gamma_{p-1, i j}=$ 0 for all $i, j \in I^{\prime}$. An absolutely relative class is of degree $k$ if $\forall i \in I^{\prime}, \sum_{j \in I^{\prime}} \gamma_{p-1,2 j}$ is an absolutely relative class of degree $k-1$ in $\mathrm{U}_{j} V_{i j}$.

2.2.3. Definition. $\gamma_{p}$ is an absolutely relative class of degree zero of $\mathrm{U}_{I^{\prime}} V_{i}$ if

(i) $0 \neq \gamma_{p, i} \in H_{p}\left(V_{i}, \bigcup_{j} V_{i j}\right)$ for all $i \in I^{\prime}$.

(ii) $\partial_{*} \gamma_{p, i}=0$ for all $i \in I^{\prime}$.

Essentially, to say a class is absolutely relative of degree $k$ means if one takes the Maier-Vietoris sequence of $\bigcup_{i \in I} V_{i}$ and breaks it up into a diagram of horizontal and vertical maps, as e.g., for $\left|I^{\prime}\right|=3$ in Diagram 1 , then $\gamma$ is of degree $k$ if one can take $k$ nonzero boundary operators on $\gamma$ before it is zero.

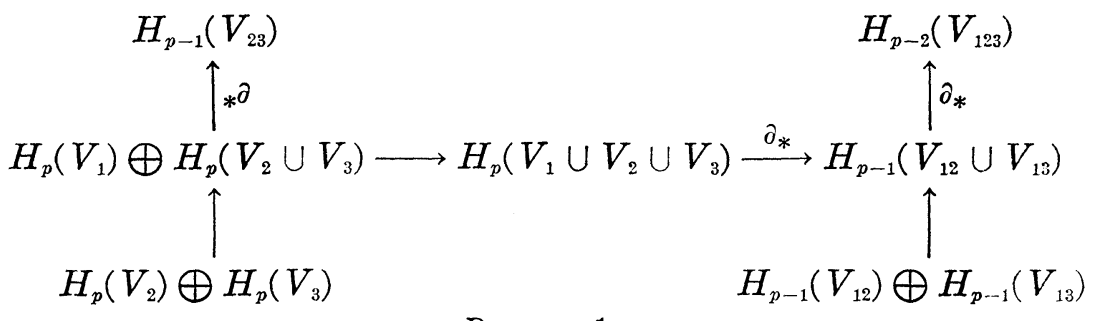

Diagram 1

Suppose $\gamma$ is a $p$-dimensional absolutely relative class of degree $k$. Then after tracing $k$ boundary operators, we have

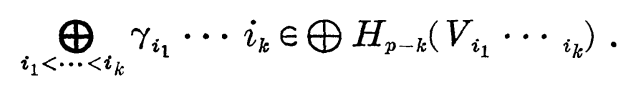

Then if $P$ denotes Poincaré duality followed by Hom, we can form $P\left(\gamma_{i_{1} \cdots i_{k}}\right) \in H_{2 n-k+p}\left(V_{i_{1}} \cdots_{i_{k}}\right)$ where $n-1=\operatorname{dim}_{c} V_{i}$.

2.2.4. Definition. $\gamma$, an absolutely relative class of degree $k$, is called tubular if

$$
\underset{i_{1}<\cdots<i_{k}}{\bigoplus_{1}} P\left(\gamma_{i_{1} \cdots i_{k}}\right) \in H_{2 n-k+p}\left(\bar{M}_{k}\right)_{\Delta} .
$$

By definition, $H_{q}\left(\bar{M}_{k}\right)_{\lrcorner} \subset H_{q}\left(\bar{M}_{k}\right)$, but in fact one easily shows 
that $H_{q}\left(\bar{M}_{k}\right)_{\Delta} \subset \bigoplus_{i_{1}<\cdots<i_{k}} H_{q}\left(V_{i_{1} \cdots i_{k}}\right)$. The mapping is given by sending $\gamma_{q}$ onto $\gamma_{q} \cap V_{i_{1} \cdots i_{k}}$ which is of dimension $q$ by (a) of the definition of tubular cycles in [4, p. 133]. It is an injection by arguments similar to those on [4, p. 135]. Hence Definition 2.2.4 is well-defined.

2.3. TheOREM. Suppose $W$ is a complex manifold of dimension $n$ and $V=\bigcup_{i \in I} V_{i}$ is a divisor with normal crossings. Let $j: W-$ $V \subset W$ be the inclusion. The then following are equivalent:

2.3.1. In compact (respectively, closed) support, the Leray spectral sequence of $j$ has $d_{k+2}^{p q}$ acting as the zero map.

2.3.2. $\tilde{\tau}_{q-k}: \widetilde{\tau}_{q-k-1} \cdots \widetilde{\tau}_{q} H_{p}\left(\bar{M}_{q}\right)_{\Delta} \rightarrow H_{p+k-1}\left(M_{q-k-1}\right)$ is an injection in compact (respectively closed) support. For $k=0, \tilde{\tau}_{q}=\tau_{q}$, and by definition, for $s \leqq 0, \widetilde{\tau}_{s}$ is always an injection.

2.3.2. Suppose $\gamma$ is an absolutely relative tubular class of degree $k$ of $\bar{M}_{q-k}\left(=\bigcup V_{i_{1} \cdots i_{q-k}}\right)$ of real dimension $2 n-2 q-p+k$ in closed (respectively compact) support. Let $f_{q-k}: \bar{M}_{q-k} \subset \bar{M}_{q-k-1}$ be the inclusion map. Then $\left(f_{q-k}\right)_{*} \gamma=0$. By definition for $s \leqq 0, f_{s}$ is always the zero map.

Proof of Theorem 2.3. The proof that (2.3.1) is equivalent to (2.3.2) follows because $d_{2}^{p, q}$ is the Gysin mapping, cf., Gordon [6, Proposition 3.3.2]. But since we are working with coefficients in a field, the vector space dual to $d_{2}^{p, q}$ acting on homology is just the transverse intersection mapping $I$. But by (2.1.2), Image $I=\operatorname{Ker} \tau$.

The equivalence of (2.3.2) and (2.3.3) is just Poincaré-Lefschetz duality, where we change supports, since if $V_{i_{1} \cdots i_{k}}$ is noncompact, then $H_{p}^{*}\left(V_{i_{1} \cdots i_{k}}, M_{k+1} \cap V_{i_{1} \cdots i_{k}}\right) \stackrel{\sim}{\rightarrow} H_{2 n-p-2 k}^{F}\left(V_{i_{1} \cdots i_{k}}-M_{k+1} \cap V_{i_{1} \cdots i_{k}}\right)$, where by closed support in $V_{i_{1} \cdots i_{k}}-M_{k+1} \cap V_{i_{1} \cdots i_{k}}$ we mean closed in $V_{i_{1} \cdots i_{k}}-$ $M_{k+1} \cap V_{i_{1} \cdots i_{k}}$ and closed in $V_{i_{1} \cdots i_{k}}$, cf., Fotiadi, et. al. [3, Part III].

For example, suppose $|I|=2$, then we have the following diagram of exact rows:

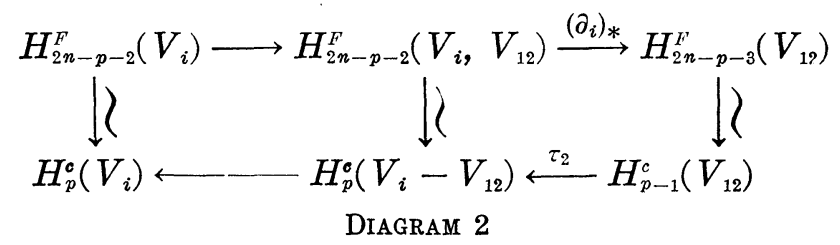

where the first row is the exact sequence of the pair $\left(V_{i}, V_{12}\right)$ and the second row follows from the Gysin sequence of the same pair. 
The vertical isomorphisms are Poincaré-Lefschetz duality followed by Hom.

Hence $\operatorname{ker} \tau_{2} \cong \operatorname{coker}\left(\partial_{i}\right)_{*}$, so that a basis of $H_{p-1}\left(V_{12}\right)$ can be chosen such that $\tau_{2}(\gamma) \neq 0$ in $V_{i}-V_{12}$ iff $P^{-1}(\gamma)=\left(\partial_{i}\right)_{*} \gamma_{i}$ for $i=1$ and 2.

Then by a similar argument, if we set $\gamma_{1}-\gamma_{2}=\gamma^{\prime} \in H_{9 n-p-2}(V)$, then $\tilde{\tau}_{1} \tilde{\tau}_{2} \gamma \neq 0$ in $W-V$ iff $\gamma^{\prime} \in \operatorname{Image}\left\{\partial_{*}: H_{2 n-p-1}(W, V) \rightarrow H_{2 n-p-2}(V)\right\}$. But $\gamma^{\prime}$ is a tubular absolutely relative class of degree one.

A similar argument works for arbitrary I.

2.4. Corollary. Suppose $W$ is a compact Kähler manifold and $V$ is a divisor with normal crossings. If $\gamma$ is an absolutely relative class of degree $k \geqq 1$ in $V$, then $i_{*} \gamma=0$ in $W$ where $i: V \subset$ $W$ is the inclusion. Thus, (2.3) gives that the spectral sequence collapses at $E_{3}$.

Corollary 2.4 follows immediately from the discussion in GriffithsSchmid [7, Chapter 4]. The key step is the principle of the two types. If we have a form $\omega$ of type $(q, p-q)$ which is exact, then $\omega=d \eta_{1}=d \eta_{2}$ where $\eta_{1}$ is of type $(q-1, p-q)$ and $\eta_{2}$ is of type $(q, p-q-1)$ [7, Lemma 2.13].

Using the principle of the two types, the idea of the proof of (2.4) is as follows: Suppose $V=V_{1} \cup V_{2}$. If $\gamma$ is an absolutely relative class of $V$, then $\gamma=\gamma_{1}+\gamma_{2}$ for $\gamma_{i} \in H_{p}\left(V_{i}\right)$ and $0 \neq \partial_{*} \gamma_{1}=-\partial_{*} \gamma_{2}=$ $\gamma_{12} \in H_{p-1}\left(V_{12}\right)$. If $i_{*} \gamma \neq 0$ for $i: V \subset W$, then there is a harmonic form $\omega$ which represents Hom $i_{*} \gamma$. We can assume $\omega$ is of pure type $(q, p-q)$ by looking at each component one by one. Then if $i_{i}: V_{i} \subset$ $W$, then $\left(i_{i}\right)^{\prime \prime} \omega$ is exact, thus by the principle of the two types, $\left(i_{i}\right)^{\prime \prime} \omega=d \eta_{1}=d \eta_{2}$. But then by Leray [9, Chapter 3], $\eta_{i} \mid V_{12}$ represents $\operatorname{Hom}\left(\gamma_{12}\right)$. Thus $\operatorname{Hom}\left(\gamma_{12}\right)$ can be represented by two different types, $(q-1, p-q)$ and $(q, p-q-1)$, which is impossible on compact Kähler manifolds. This is also what happens in the lemma of the two filtrations of Deligne $[2,1.3]$.

2.5. We note that (2.3) is a topological and not an analytic fact. That is, suppose $\omega$ is a orientable, $C^{\infty}$ manifold and $V=\mathrm{U}_{i} V_{i}$ is a collection of $C^{\infty}$ submanifolds in general position. Then (2.1) can be modified so that (2.1.2) is still true, cf., Gordon [5], and (2.2) still makes sense. Then (2.3) will still be true with appropriate modification in the indices of (2.3.2) and (2.3.3) which will only depend on the codimension of the $V_{i}$.

In particular, suppose $W$ is a compact Kähler manifold and $V=$ $\bigcup_{i} V_{i}$ are complex submanifolds in general position. Let $N=\max _{i} n_{i}$ where $n_{i}$ is the complex codimension of $V_{i}$. Then (2.4) actually shows 
that the Leray spectral sequence of $j: W-V \subset W$ collapses at $E_{2 N+1}$.

However, it is easy to give topological examples where the spectral sequence does not degenerate at the approprimate codimension. E.g., let $S_{1}$ and $S_{2}$ be two 2-dimensional spheres in $R^{4}$ which intersect transversely in two points $P$ and $Q$. Let $T(S)$ be a regular tubular neighborhood of $S=S_{1} \cup S_{2}$ in $R^{4}$ and form $D T(S)$, the double of $T(S)$, by glueing $T(S)$ to itself along its boundary. Then $D T(S)$ is a compact 4-dimemsional manifold and $S$ is two submanifolds in general position of real codimension two.

Let $\gamma_{1}$ be the one cycle formed by tracing a line from $P$ to $Q$ in each of the $S_{i}$. Then $0 \neq\left[\gamma_{1}\right] \in H_{1}(S)$. If $i: S \subset D T(S)$, then it is easy to see that $i_{*}\left[\gamma_{1}\right] \neq 0$ in $D T(S)$. Hence by (2.3.3), the Leray sequence of $j: D T(S)-S \subset D T(S)$ does not degenerate at $E_{3}^{0,2} \neq E_{4}^{0,2}$, but at $E_{4}^{p, q} \cong E_{\infty}^{p, q}$.

The obvious examples one would consider for $W$ a compact nonKähler surface do not yield examples where the spectral sequence collapses at $E_{3}$. For example, if $W$ is an elliptic surface with singular fibre of type ${ }_{m} I_{b}$ with $b>1$, cf. Kodaira [10], then the spectral sequence associated to this singular fibre always degenerates at $E_{3}$, i.e., the cycle formed by joining all the double points always bounds in $W$. If such an example exists for surfaces, it would probably have to be of type VII with $b_{1} \equiv 1(2)$ and $b_{2}>0$.

\section{Arbitrary singularities in the compact Kähler case.}

3.1. If $V \subset W$ is a variety of complex codimension $N$ and $W$ is a complex manifold, then one can resolve the singularities of $V$ by a finite number of monoidal transforms with nonsingular centers, cf., Hironaka [8]. That is, one has another complex manifold $W^{\prime}$ and a holomorphic map $\pi: W^{\prime} \rightarrow W$ such that if $\pi^{-1}(V)=V^{\prime}$, then $V^{\prime}$ is a union of complex submanifolds in general position with $\operatorname{codim}_{c} V^{\prime}=$ $N$. Furthemore, by a theorem of Blanchard [1], if $W$ is compact Kähler, then so is $W^{\prime}$.

Then one has two spectral sequences with regards to the two maps $j: W-V \subset W, j^{\prime}: W^{\prime}-V^{\prime} \subset W^{\prime}$ with $E_{2}^{p q} \Rightarrow H^{p+q}(W-V)$ and ${ }^{\prime} E_{2}^{p q} \Rightarrow H^{p+q}\left(W^{\prime}-V^{\prime}\right)$. Now, by the general theory of monoidal transforms one has that $\pi$ is a bianalytic homeomorphism of $W-V$ onto $W^{\prime}-V^{\prime}$. Furthermore, by [5, p. 56] and by [4, Proposition 3.1], we have the following:

3.1.1. Proposition. $\operatorname{Im} E_{\infty}^{p, q} \stackrel{\sim}{\rightarrow} \operatorname{ker}\left\{H_{p+q}(W-V) \rightarrow H_{p+q}(W)\right\}$ and $\operatorname{Im} E_{\infty}^{p, q} \cong \operatorname{Im}{ }^{\prime} E_{\infty}^{p, q}$ under the identification $\pi: W-V \stackrel{\sim}{\rightarrow} W^{\prime}-V^{\prime}$. 
3.2. Of course, if $V$ is a variety of complex codimension $N$ whose singular locus is of sufficiently high codimension in $V$, then then spectral sequence will not collapse at $E_{2 N+1}$, even if $W$ is compact Kähler.

For example, if in $C P^{3}$, we let $C(T)$ be the cone over the torus in $C P^{2}$, i.e., $C(T)=\left\{[x, y, z, w] \mid x^{3}+y^{3}+z^{3}=0\right\}$, then $E_{2}^{4,1} \cong H^{4}(C(T))$ and $E_{2}^{0,4} \cong H^{3}(L)$, where $L$ is the intersection of $C(T)$ and a sufficiently small 5-sphere about the singular point of $C(T)$. This follows because $E_{2}^{p q} \cong H^{p}\left(W ; R^{q}\right)$, where $R^{q}$ is the sheaf associated to the presheaf which sends the open set $U \rightarrow H^{q}(U-V \cap U)$, cf., Swan [11, p. 129]. Hence, $d_{4}^{0,4}: E_{4}^{0,4}=E_{2}^{0,4} \rightarrow E_{4}^{4,1}=E_{2}^{4,1}$ is dual to the intersection mapping of $H_{4}(C(T))$ onto $H_{3}(L)$, which is nonzero, i.e., $E_{5}^{0,4}=0$.

But, when we do not have normal crossings, even in the compact Kähler case, we can have counterexamples to (2.3.2). For example, let $T$ be the torus in $C(T)$ as above, and let $W$ be the projective, algebraic manifold gotten by a monoidal transform $\pi$ in $C P^{3}$ with center $T$. Let $V \subset W$ be the strict transform of $C(T)$ by $\pi$. Then $V \cong C(T)$, since $T$ is of codimension one in $C(T)$. Hence $V$ has one singular point, which we shall call $P_{\infty}$; and a Whitney stratification of $V$ is given by $M_{1}=V-P_{\infty}, M_{2}=P_{\infty}$.

Consider $[\gamma] \in H_{1}(T), T \subset V$. Then $\gamma \sim 0$ in $V$; in fact $\gamma=\tau_{2}\left(P_{\infty}\right)$ where $\tau_{2}: H_{*}\left(M_{2}\right) \rightarrow H_{*}\left(M_{1}\right)$ is the tube over cycle map, [4, p. 158]. Hence, $0 \neq[\gamma] \in E_{2}^{0,2}$. But $\tau_{1} \tau_{2} P_{\infty}=0$ in $W-V$, since there is a $\left[\gamma_{3}\right] \in$ $H_{3}(W)$ such that $\gamma_{3} \cap V=\gamma$. Topologically, $\gamma_{3}=\pi^{-1}(\gamma)$. Here $E_{4}^{0,2} \cong$ $E_{\infty}^{02}$.

Similarly, taking $V_{m}=\underbrace{V \times \cdots \times}_{m \text { times }} \times V \subset \underbrace{W \times \cdots \times}_{m \text { times }} W=W_{m}$, we can form a Whitney stratification of $V_{m}$ by letting $M_{1}$ be the smooth points of $V_{m}, M_{2}$ be the smooth points of $V_{m}-M_{1}, M_{3}$ be the smooth points of $V_{m}-M_{1} \cup M_{2}$, etc., so that $M_{m+1}=P_{\infty} \times \cdots \times P_{\infty}=P$. Then $\tau_{2} \cdots \tau_{m+1}(P) \ngtr 0$ in $M_{1}$ (topologically, it is $\gamma \times \cdots \times \gamma$ ), where $\tau_{i}$ are the iterated tubes maps defined in Gordon [4, p. 158]. But $\tau_{1} \tau_{2} \cdots \tau_{m+1}(P) \sim 0$ in $W_{m}-V_{m}$, i.e., $d_{3 m}^{0,3 m-1}$ is not the zero map.

What happens that we have a product of circle each of which bounds a disk in $V$, but when one resolves the singularities, this product of circle defines a nonzero homology class in $V^{\prime}$. What (2.3.2) states is that when the ambient space is compact Kähler this always happens:

3.2.1. Proposition. Let $W$ be a compact Kähler manifold and $V$ an arbitrary variety. If an iterated tube map is not injective, then when one resolves the singularties via nonsingular centers, those cycles (in the kernel of the iterated tube map) represent nonzero homology classes in the proper transform. 


\section{REFERENCES}

1. A. Blanchard, Sur les variétes analytiques complexes, Ann. Ec. Norm (3), 73 (1956), 157-202.

2. P. Deligne, Theorie de Hodge II, IHES Publications Mathématiques, 40 (1972), 5-57.

3. D. Fotiadi, M. Froissard, J. Lascoux and F. Pham, Applications of an isotropy theorem, Topology, 4 (1964), 159-191.

4. G. L. Gordon, The residue calculus in several complex variables, Trans Amer. Math. Soc., 213 (1975), 127-176.

5. — $A$ Poincare' duality type theorem for polyhedra, Ann. Inst. Fourier (Grenoble), 22 (1972), 47-58.

6. — Differentials of the second kind (preprint), 1976.

7. P. A. Griffiths and W. Schmid, Recent.Developments in Hodge Theory, mimeographed notes from Tata Institute.

8. H. Hironaka, Bimeromorphic mappings, mimeographed from Warwick University, 1971.

9. J. Leray, Cauchy Probleme III, Bull. Soc. Math. France, 87 (1959), 81-180.

10. K. Kodaira, On compact analytic surfaces II, Annals of Math., 77, no. 3 (1963), $563-626$.

11. R. Swan, The Theory of Sheaves, Un. of Chicago Press, Chicago, 1964.

Received July 10, 1978. This work was partially supported by NSF contract GP 38964A \#1.

DePaUl University

Chicago, IL 60614 


\section{PACIFIC JOURNAL OF MATHEMATICS}

\section{EDITORS}

DONALD BABBITT (Managing Editor)

University of California

Los Angeles, CA 90024

Hugo RossI

University of Utah

Salt Lake City, UT 84112

C. C. MOORE and ANDREW OGG

University of California

Berkeley, CA 94720

\section{J. DugunduI}

Department of Mathematics

University of Southern California

Los Angeles, CA 90007

R. Finn and J. Milgram

Stanford University

Stanford, CA 94305

\section{ASSOCIATE EDITORS}
E. F. BeCKenBACH
B. H. NeumanN
F. WOLF
K. YosHIDA

\section{SUPPORTING INSTITUTIONS}

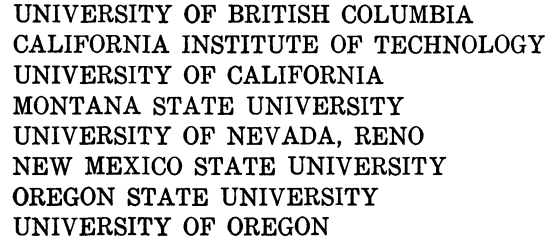

UNIVERSITY OF BRITISH COLUMBIA CALIFORNIA INSTITUTE OF TECHNOLOGY UNIVERSITY OF CALIFORNIA

MONTANA STATE UNIVERSITY

UNIVERSITY OF NEVADA, RENO

NEW MEXICO STATE UNIVERSITY

OREGON STATE UNIVERSITY UNIVERSITY OF OREGON

\author{
UNIVERSITY OF SOUTHERN CALIFORNIA \\ STANFORD UNIVERSITY \\ UNIVERSITY OF HAWAII \\ UNIVERSITY OF TOKYO \\ UNIVERSITY OF UTAH \\ WASHINGTON STATE UNIVERSITY \\ UNIVERSITY OF WASHINGTON
}

The Supporting Institutions listed above contribute to the cost of publication of this Journal, but they are not owners or publishers and have no responsibility for its content or policies.

Mathematical papers intended for publication in the Pacific Journal of Mathematics should be in typed form or offset-reproduced, (not dittoed), double spaced with large margins. Please do not use built up fractions in the text of the manuscript. However, you may use them in the displayed equations. Underline Greek letters in red, German in green, and script in blue. The first paragraph or two must be capable of being used separately as a synopsis of the entire paper. Please propose a heading for the odd numbered pages of less than 35 characters. Manuscripts, in triplicate, may be sent to any one of the editors. Please classify according to the scheme of Math. Reviews, Index to Vol. 39. Supply name and address of author to whom proofs should be sent. All other communications should be addressed to the managing editor, or Elaine Barth, University of California, Los Angeles, California, 90024.

50 reprints to each author are provided free for each article, only if page charges have been substantially paid. Additional copies may be obtained at cost in multiples of 50 .

The Pacific Journal of Mathematics is issued monthly as of January 1966. Regular subscription rate: $\$ 84.00$ a year (6 Vols., 12 issues). Special rato: $\$ 42.00$ a year to individual members of supporting institutions.

Subscriptions, orders for numbers issued in the last three calendar years, and changes of address shoud be sent to Pacific Journal of Mathematics, P.O. Box 969, Carmel Valley, CA 93924, U.S.A Old back numbers obtainable from Kraus Periodicals Co., Route 100, Millwood, NY 10546.

PUBLISHED BY PACIFIC JOURNAL OF MATHEMATICS, A NON-PROFIT CORPORATION

Printed at Kokusai Bunken Insatsusha (International Academic Printing Co., Ltd.). 8-8, 3-chome, Takadanobaba, Shinjuku-ku, Tokyo 160, Japan.

Copyright (C) 1980 by Pacific Jounal of Mathematics Manufactured and first issued in Japan 


\section{Pacific Journal of Mathematics \\ Vol. 90, No. $2 \quad$ October, 1980}

Frank Hayne Beatrous, Jr., Hölder estimates for the $\bar{\partial}$ equation with a support condition ..................................... 249

Charles L. Belna, Michael Jon Evans and Paul Humke, Planar continua

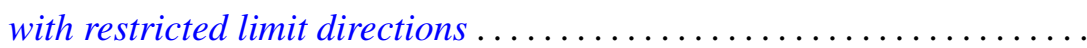

Leon Brown and Takashi Ito, Classes of Banach spaces with unique isometric preduals................................. 261

V. K. Deshpande, Completions of Noetherian hereditary prime rings ..... . 285

Deepak Dhar, Asymptotic enumeration of partially ordered sets . . . . . . . 299

Zeev Ditzian, On interpolation of $L_{p}[a, b]$ and weighted Sobolev

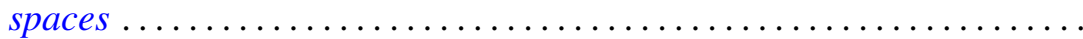

Andrew George Earnest, Congruence conditions on integers represented by

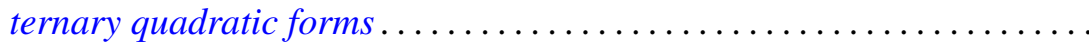

Melvin Faierman, Bounds for the eigenfunctions of a two-parameter system of ordinary differential equations of the second order ..............

Hector O. Fattorini, Vector-valued distributions having a smooth

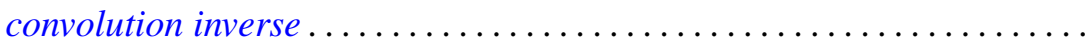

Howard D. Fegan, The spectrum of the Laplacian on forms over a Lie

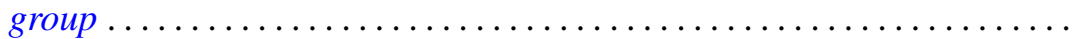

Gerald Leonard Gordon, On the degeneracy of a spectral sequence associated to normal crossings..

S. Madhavan, On bisimple weakly inverse semigroups ... 397

Françoise Mathot, On the decomposition of states of some

Roger McCann, Embedding asymptotically stable dynamical systems into radial flows in $l_{2}$

Michael L. Mihalik, Ends of fundamental groups in shape and proper homotopy...............................

Samuel Murray Rankin, III, Boundary value problems for partial functional differential equations .

Randy Tuler, Arithmetic sums that determine linear characters on $\Gamma(N)$

Jeffrey D. Vaaler, On linear forms and Diophantine approximation ..

G. P. Wene, Alternative rings whose symmetric elements are nilpotent or a right multiple is a symmetric idempotent. . 